\title{
Integração de um sistema fotovoltaico isolado e de coleta seletiva de resíduos em um quiosque multifuncional
}

\author{
Fabiano Almeida Nascimento ${ }^{1}$, Samara Pereira Vieira ${ }^{1}$, Sizenando José \\ de Andrade Júnior ${ }^{2}$, Luiz Antônio Pimentel Cavalcanti²**
}

${ }^{1}$ Instituto Federal de Educação, Ciência e Tecnologia da Bahia, Campus de Paulo Afonso, Rua Marcondes Ferraz, 200, Bairro General Dutra, Paulo Afonso-BA (CEP 48607-000). Estudante de Graduação em Engenharia Elétrica.

${ }^{2}$ Instituto Federal de Educação, Ciência e Tecnologia da Bahia, Campus de Paulo Afonso, Rua Marcondes Ferraz, 200, Bairro General Dutra, Paulo Afonso-BA (CEP 48607-000). Professor do Ensino Básico, Técnico e Tecnológico (EBTT). "E-mail: luizufpe@yahoo.com.br; luiz.cavalcanti@ifba.edu.br.

Resumo. A revolução da matriz energética mundial perpassa pela introdução de novas fontes de geração de energia elétrica visando a reduzir os danos à Natureza e o menor custo de geração em relação às fontes convencionais. Nesse contexto, o presente trabalho tem como objetivo atender parte da demanda energética da comunidade acadêmica local, utilizando um sistema fotovoltaico no intuito também de promover coleta seletiva e conscientização sobre sustentabilidade. Para tanto é disponibilizado uma central de recarga de aparelhos eletrônicos pessoais, como celulares, tablets e notebooks, através de cabos multi-USB e tomadas $2 \mathrm{P}+\mathrm{T}$, que disponibilizam energia elétrica proveniente da fonte solar, incluindo em seu arranjo espaços para coleta seletiva de baterias, plásticos, metais, papeis e vidros. O projeto almeja alocar protótipos em instituições de ensino visando a incrementar a didática de aprendizado sobre o funcionamento da geração elétrica de energia, através do recurso solar e, paralelamente, incentivar a criação de uma cultura voltada à utilização de fontes renováveis não convencionais na matriz energética brasileira. $\mathrm{O}$ primeiro protótipo do projeto foi desenvolvido e instalado no Instituto Federal de Educação, Ciência e Tecnologia da Bahia (IFBA), Campus de Paulo Afonso, e tem sido utilizado amplamente pela comunidade acadêmica para recarga de equipamentos e entretenimento, bem como tem dado suporte a pesquisas científicas com ênfase em energias renováveis e coleta seletiva de resíduos.

Palavras-chave: Sustentabilidade, Energia Fotovoltaica, Coleta Seletiva.

\begin{abstract}
Integration of an isolated photovoltaic system with selective waste collection in a multifuncional kiosk. The change in global energy matrix has permeated by the introduction of new electric energy sources aiming a lower production cost and decreasing of environmental damage, when observed conventional sources of generation. In this context, the present project aims to provide part of the electric demand of local academic community, using a photovoltaic system and in order to promote selective collection of waste and awareness about sustainability. By this way is available a personal central for recharging of electronic devices as mobile

Recebido: 21/05/2015

Aceito: 03/06/2015

Publicado: 30/06/2015

Acesso Aberto

Artigo completo

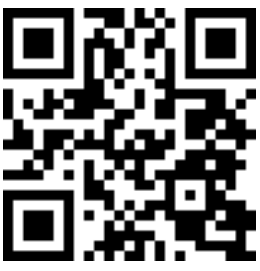


phones, tablets and laptops, through Multi-USB cables and sockets $2 \mathrm{P}+\mathrm{T}$ that provides electricity from the solar source, including in its scratch spaces to selective waste collection of batteries, plastics, metals, papers and glass. The project goal is to allocate prototypes in education institutions targeting improving the teaching of the power generation through solar resource and, in parallel, encourage the creation of a culture in using unconventional renewable sources of energy in Brazilian energy matrix. The first prototype of this project was developed and installed in the Federal Institute of Education, Science and Technology of Bahia, Paulo Afonso Campus, and it has been widely used by the academic community for recharging electronic equipment and as entertainment. It has given support to scientific research with emphasis on renewable energy and selective waste collection.

Keywords: Sustainability, Photovoltaic energy, Waste selective collection.

\section{Introdução}

O crescimento da população mundial, associado ao desenvolvimento tecnológico e industrial, conduz a um aumento substancial de demanda energética e as fontes de energia hoje utilizadas são provenientes de recursos naturais limitados e poderão se esgotar em algumas décadas (Dunham e Iverson, 2014). De maneira que a disponibilidade de energia não mais corresponde à demanda imposta pela estrutura espacial da vida urbana, o que implica num período de adaptação onde se evidencia um período de redução de possibilidades energéticas, principalmente das originadas do petróleo, que é uma fonte de energia polivalente. Sendo que as fontes de energia nucleares, geotérmicas, solares, biomassa e outras, que substituirão as atuais, deverão, nas próximas décadas, ajustarem-se às necessidades de utilizações, para que não venham a causar problemas ambientais (Silva et al., 2014).

Uma das grandes apostas para resolução deste problema energético é a conversão direta da energia solar em fenômeno intermediado por placas solares, que se caracterizam por células fotovoltaicas associadas eletricamente, conseguindo disponibilizar uma carga elétrica com a exposição do sistema ao sol. Atualmente, os sistemas que consistem em gerar energia elétrica a partir do efeito fotovoltaico armazenando-a em baterias ou depositando-a na rede, são muito utilizados, porém com propósito de alimentação residencial ou industrial, sendo estes sistemas robustos, com alto valor pecuniário e de difícil aplicação em locais públicos (Bittencourt e Bassaco, 2013).

Em relação às questões ambientais atuais, a crescente demanda energética não é o único problema que necessita de atenção urgente. $\mathrm{O}$ correto direcionamento dos resíduos sólidos mostra-se como um grande desafio a ser encarado e debatido, sendo necessário amplo processo educacional (Santos e Soares, 2014).

Segundo a Associação Brasileira de Empresas de Limpeza Pública e Resíduos Especiais - Abrelp (2013), a geração de resíduos sólidos urbanos no Brasil em 2013 foi de $76.387 .200 \mathrm{t}$, o que representa um aumento de 4,1\% em relação ao ano de 2012, superando a taxa de crescimento populacional, que foi de 3,7\%. Em consonância, a coleta seletiva de resíduos cresceu 4,4\% no comparativo 2012/2013, com um total de 69.064.935 t coletadas em 2013. Entretanto este dado não é animador, pois quando observado a quantidade de resíduos sólidos urbanos gerados e coletados no ano de 2013, observa-se que diariamente $20.000 \mathrm{t}$ deixaram de ser coletadas no país e, por consequência, tiveram destino impróprio (Abrelp, 2013).

Ainda de acordo com a Abrelp (2013), não foi identificado um crescimento considerável nas iniciativas de municípios perante a coleta seletiva, sendo que, em 2012, 60,2\% dos municípios brasileiros apresentaram ações para coleta seletiva e $62 \%$ no ano de 2013. Observa-se que de 
acordo com a Política Nacional de Resíduos Sólidos (PNRS), a gestão e gerenciamento de resíduos sólidos urbanos devem ser orientados pelas seguintes prioridades: não geração, redução, reutilização, reciclagem, tratamento de resíduos sólidos e disposição final ambientalmente adequada. Todos esses itens têm por objetivo mitigar os impactos ambientais causados pelos resíduos ao meio ambiente, reduzir a carga de resíduos disposta nos aterros sanitários aumentando sua vida útil, além de reinserilos na cadeia produtiva por meio da reciclagem, gerando emprego e renda (Paschoalin Filho et al., 2014).

Analisando o crescente hábito de utilização de dispositivos eletrônicos pessoais, observa-se, como consequência, a crescente necessidade de armazenamento de energia elétrica através de baterias. Em termos de carga elétrica, as baterias utilizadas em dispositivos eletrônicos pessoais não apresentam autonomia suficiente para as atividades corriqueiras, aumentando assim a periodicidade de recargas diárias. Quando associados, o enraizamento de culturas voltadas à utilização dos dispositivos eletrônicos, a indisponibilidade de pontos de recarga em locais públicos e a baixa autonomia das baterias atualmente utilizadas, apresentamse como problema a ser resolvido (Silva, 2010).

Visto que a disponibilidade de energia elétrica e de pontos de coleta de resíduos sólidos não vem sendo ofertadas de forma ampla e abrangente, o presente trabalho trata da prototipagem e instalação de uma central de recarga de dispositivos eletrônicos pessoais à base de energia solar, em conjunto com um sistema de coleta seletiva de resíduos sólidos.

Tendo como objetivo ofertar energia elétrica a partir de um sistema gerador baseado em células fotovoltaicas, desligadas da rede, visando à disponibilização de pontos de entrega de energia elétrica aos usuários de dispositivos eletrônicos pessoais, para recarga, além de acolher de forma seletiva os resíduos que ora venham a ser despejados nos seus coletores. O protótipo foi alocado nas instalações do Instituto Federal de Educação Ciência e Tecnologia da Bahia (IFBA), Campus de Paulo Afonso.
Objetiva-se ainda, este estudo, disponibilizar o protótipo para pesquisas acadêmicas voltadas à utilização de energia fotovoltaica desconectada da rede, para estudos probabilísticos, estatísticos e energéticos, além de estudos relativos à coleta seletiva de resíduos. Pretende-se, paralelamente, inserir na instituição uma cultura de práticas renováveis e a percepção da utilização direta de uma energia elétrica renovável de fonte não convencional, diminuindo assim a intangibilidade com esta tecnologia.

\section{Materiais e métodos}

Inicialmente, para projetar estruturalmente o protótipo, utilizou-se o software Sketchup ${ }^{\circledR}$, versão 2014 (Sketchup, 2014). Finalizado a estrutura básica do desenho com os requisitos objetivados, iniciou-se a análise orçamentária e decisão dos conjuntos de materiais que deveriam ser utilizados. Paralelamente, partindo do objetivo da disponibilidade de 1,88 Ah/dia, valor condizente para atendimento do público alvo em termos de recarga de dispositivos eletrônicos, dimensionou-se os condutores, bateria, inversor de frequência, controlador de carga e placa fotovoltaica seguindo as normatizações vigentes e observando a qualidade dos materiais em consonância com sua vida útil.

A média da irradiação solar incidente foi obtida mensalmente na estação solar de Paulo Afonso e os dados organizados sistematicamente de modo a apontar a potência teórica a ser gerada e a inclinação ideal do módulo fotovoltaico (Tabela 1).

Obtidos os materiais e dispositivos eletroeletrônicos necessários, foi finalizada a estrutura física projetada e iniciou-se o processo de montagem e acabamento do protótipo. As sinalizações, bem como os detalhes estéticos e estruturais foram projetados e inseridos na conclusão do processo de integração da parte elétrica e estrutural. Com o protótipo pronto, iniciouse o processo de montagem nas instalações do IFBA, Campus de Paulo Afonso, com o intuito da realização de testes. Em um primeiro momento, o seu uso foi restrito à equipe do projeto, para que testes de curto 
Tabela 1. Irradiação solar diária média mensal de acordo com a Estação Solar de Paulo Afonso (Cresesb).

\begin{tabular}{lcccccccccccccc}
\hline \multirow{2}{*}{ Ângulo } & \multirow{2}{*}{ Inclinação } & Jan & Fev & Mar & Abr & Maio & Jun & Jul & Ago & Set & Out & Nov & Dez & Média \\
\hline PH & $0^{\circ} \mathrm{N}$ & 5,78 & 5,67 & 5,69 & 5,03 & 4,36 & 4,06 & 4,31 & 5,25 & 5,44 & 6,36 & 6,50 & 5,38 & 5,38 \\
AIL & $9^{\circ} \mathrm{N}$ & 5,49 & 5,51 & 5,70 & 5,21 & 4,64 & 4,38 & 4,63 & 5,52 & 5,52 & 6,23 & 6,19 & 5,40 & 5,40 \\
MMA & $6^{\circ} \mathrm{N}$ & 5,60 & 5,57 & 5,71 & 5,16 & 4,56 & 4,28 & 4,53 & 5,44 & 5,50 & 6,29 & 6,30 & 5,41 & 5,41 \\
MMM & $25^{\circ} \mathrm{N}$ & 4,77 & 5,01 & 5,46 & 5,29 & 4,93 & 4,76 & 4,99 & 5,75 & 5,41 & 5,73 & 5,39 & 4,96 & 5,20 \\
\hline
\end{tabular}

PH = Plano horizontal; AIL = Ângulo igual à latitude; MMA = Maior média anual; MMM = Maior mínimo mensal.

circuito, sobrecarga, sobtensão e subtensão, pudessem ser realizados. Ao fim da instalação e sessão de testes, o protótipo teve seu uso liberado para toda comunidade.

Após o processo de dimensionamento dos dispositivos eletroeletrônicos do protótipo, seguiu-se o processo orçamentário até que procedemos com a compra dos equipamentos, que foram analisados como o melhor custo e benefício visando aos critérios da qualidade, vida útil, garantia disponibilidade de venda na região e normatização. Foram adquiridos em loja de eletrônicos da Região de Paulo Afonso: a) Inversor de frequência: Inversor de onda modificada Hayonik, $400 \mathrm{~W} 12 \mathrm{~V} / 127 \mathrm{~V}$; b) Controlador de carga CMTP02 $12 \mathrm{~V}$ e $10 \mathrm{~A}$; c) Placa Solar: Golden Genesis PV-110E - $110 \mathrm{Wp}$; e d) Bateria: Tudor estacionária 45 Ah.

\section{Resultados e discussão}

De acordo com os dados colhidos através da Estação Solar de Paulo Afonso, do Centro de Referência para Energia Eólica e Solar (Cresesb), no ano de 2014, a cidade apresentou irradiação solar média diária, para plano inclinado em ângulo igual à latitude (inclinação ideal para dispo sição do módulo fotovoltaico), de 5,40 kWh.m ${ }^{-2} \cdot$ diaa $^{-1}$ (Tabela 1), indicando uma grande atratividade energética de aplicação do sistema por apresentar um valor acima da média geral do Brasil.

Conforme planejamento inicial deste projeto, toda a construção do protótipo foi desenvolvida a partir de modelagem, usando o Sketchup ${ }^{\circledR} 2014$ (Sketchup, 2014). Em consonância com os objetivos e prevendo a utilização pessoal do protótipo, projeções ergonômicas foram pensadas e incluídas no desenho. O design do projeto deveria chamar atenção e ter arquitetura inovadora, visto que ele deve ser alvo de curiosidade perante seu público. A Figura 1 aponta o desenho desenvolvido no software e usado na construção do protótipo.

Já com os dispositivos citados e a estrutura física central em mãos, procedeuse a montagem dos elementos de circuito elétrico do protótipo, que se deu sem problemas e em poucos dias diante da execução do planejamento explicitado na metodologia (Figura 2).

Com a finalização de todo o sistema elétrico, partiu-se para o acabamento da peça central (Figura 3).

Observando a norma de segurança NR-10/2004, do Ministério do Trabalho e Emprego, determinada para instalação mecânica e elétrica de baixa tensão, procedeu-se com a acomodação do circuito na parte central do protótipo, dispondo dos equipamentos e materiais como projetado. O posicionamento do módulo fotovoltaico respeitou a altura mínima de obstáculos presentes ao seu redor, visto que o local planejado para alocação contem árvores que poderiam atrapalhar a incidência solar durante os períodos de nascer e pôr do sol, assim ficando a uma altura de $3,25 \mathrm{~m}$ do nível do solo e $2 \mathrm{~m}$ do nível máximos da copa das árvores.

O módulo fotovoltaico foi disposto na direção norte/sul alinhado ao seu lado de maior comprimento, com inclinação para o norte em $15^{\circ}$ visando a ampliar os ganhos em geração de energia elétrica. Ainda no intuito de melhorar a eficiência do sistema, em detrimento da angulação de $9^{\circ}$ (ângulo igual à latitude), utilizou-se uma defasagem angular de $15^{\circ}$ em relação ao eixo imaginário paralelo ao nível do solo, 


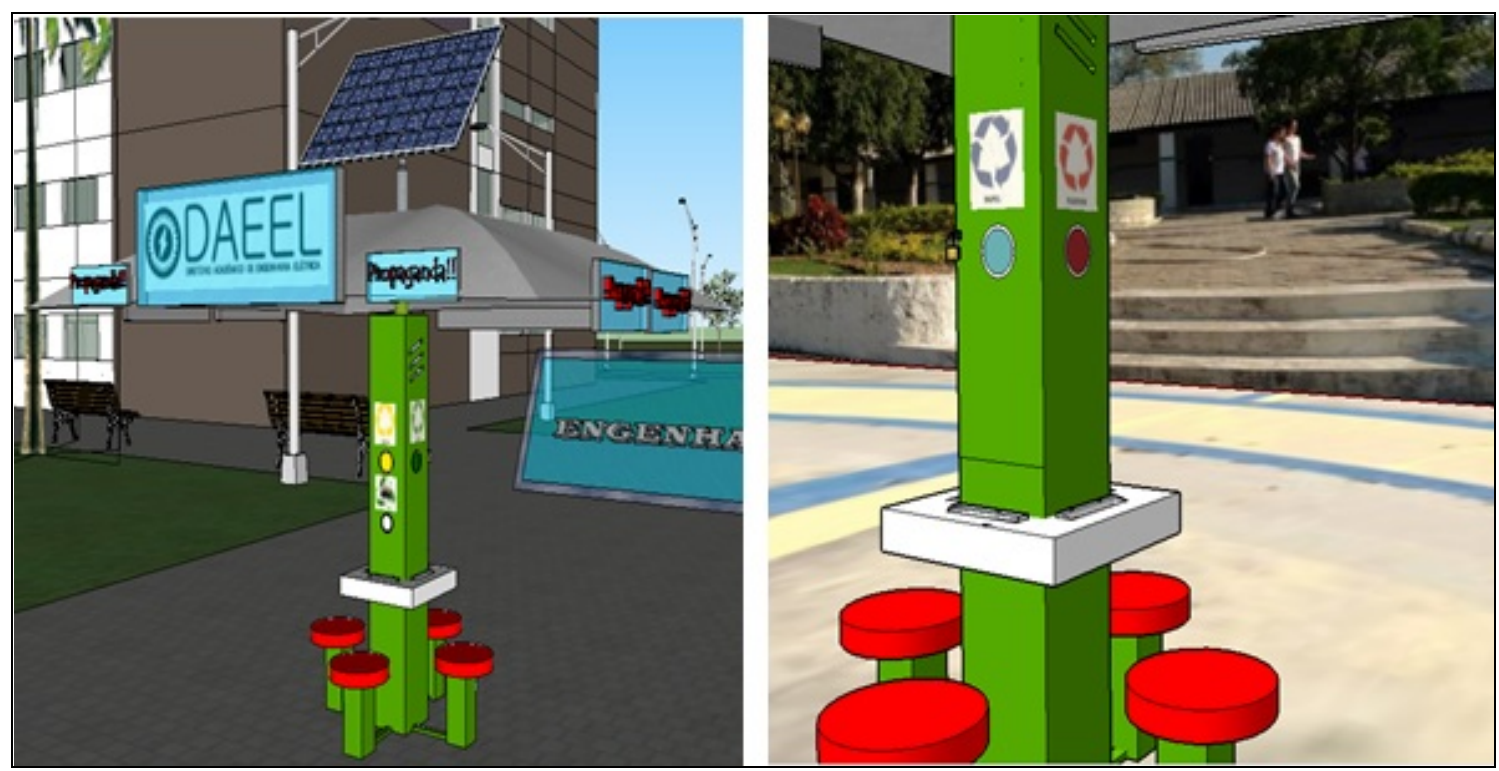

Figura 1. Desenho em software Sketchup ${ }^{\circledR}$, mostrando a vista humana do protótipo disposto nas instalações do IFBA, Campus de Paulo Afonso.

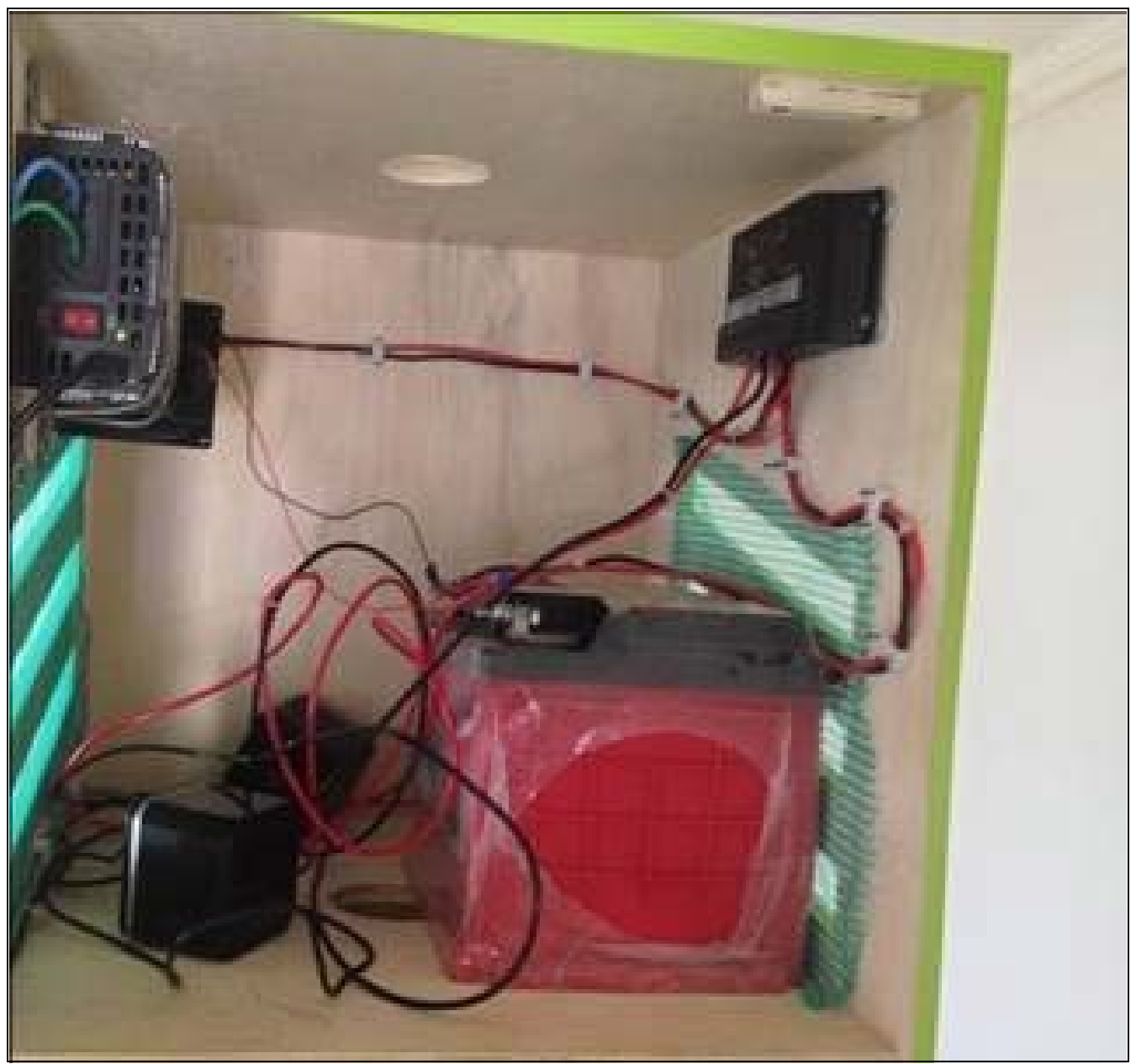

Figura 2. Disposição dos dispositivos eletrônicos no interior da peça central. 

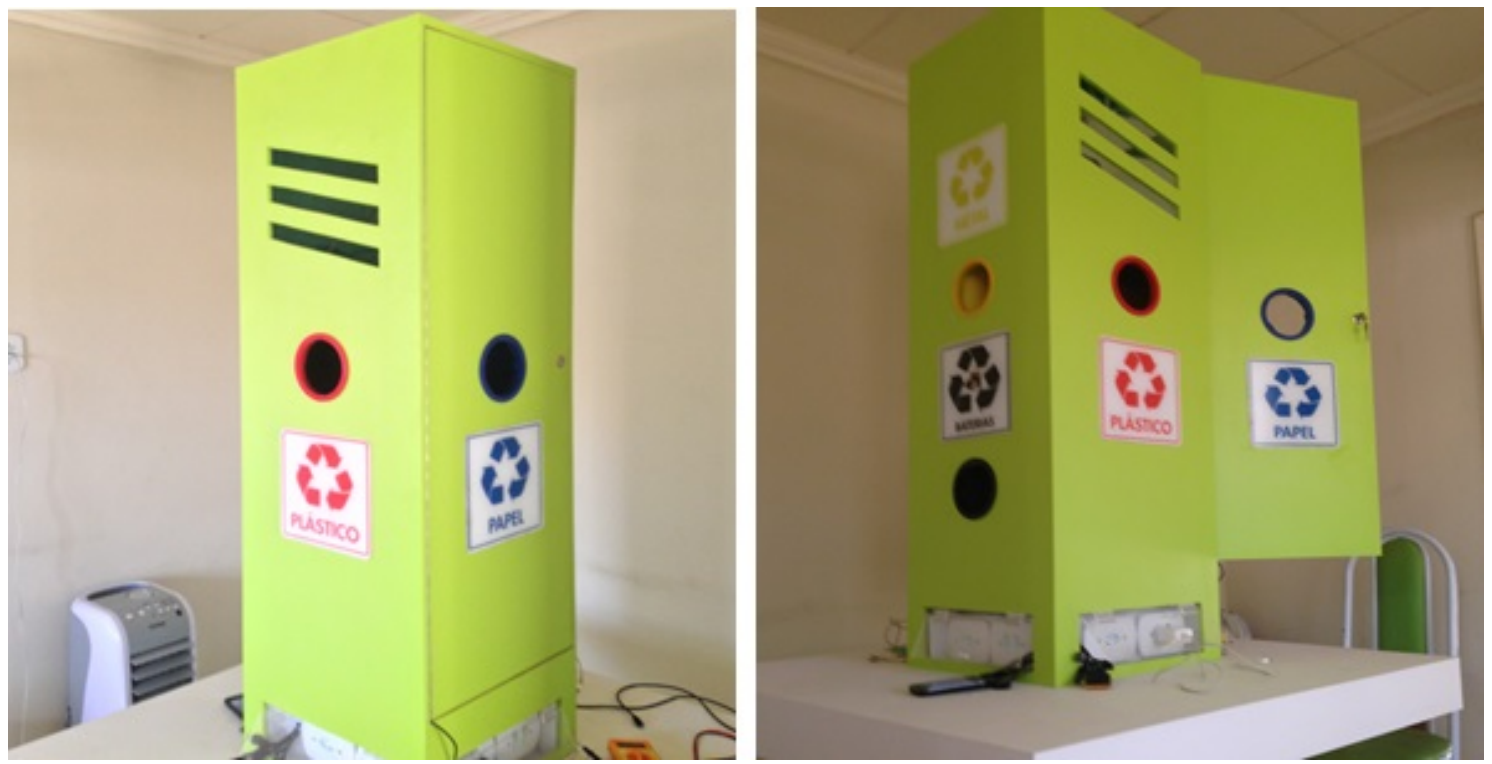

Figura 3. Parte central do protótipo com acabamento finalizado.

buscando evitar perdas de geração relacionadas ao estacionamento de corpos estranhos (folhas, poeiras, fuligem, papeis, etc.) na superfície do módulo fotovoltaico.

O sistema fotovoltaico implementado ao protótipo possui uma capacidade de geração diária, observado os índices de irradiação solar local de aproximadamente 660 Wh. Como o sistema de geração apresenta tensão e corrente continua mantendo-se seus valores nominais de $16,7 \mathrm{~V}$ e 6,6 A respectivamente, conclui-se que em um dia de plena carga, em condições meteorológicas favoráveis, a bateria armazenaria uma quantia média de $40 \mathrm{Ah}$, completando sua capacidade nominal. Essa quantidade de energia armazenada é capaz de disponibilizar, a um único aparelho celular, carga durante $40 \mathrm{~h}$ (considerando fontes carregadores de $5 \mathrm{~V}$ e 1 A ou ainda manter carregando constantemente um notebook por $13 \mathrm{~h}$ ). Sabido que o ciclo noturno, quando não há geração de energia elétrica, dura em média $12 \mathrm{~h}$, considera-se que o dimensionamento feito atende a necessidade demandada.

Em termos práticos, foi observado que o protótipo é utilizado diariamente por 30 pessoas, em média. As cargas variam de acordo com o usuário, o tipo de aparelho que desejam carregar e o tempo de utilização. Por ser um ambiente aconchegante e descontraído, os lugares destinados à utilização do protótipo estão constantemente ocupados, assim, devido à boa aceitação do público acadêmico ao uso do protótipo, obstinasse que sejam postos mais de uma unidade na instituição, com o intuito de atender o público de forma mais abrangente.

A Figura 4 mostra o protótipo pronto e instalado no IFBA. O uso está se dando de forma constante pelos discentes, docentes, técnico-administrativos e terceirizados do Campus.

O protótipo reagiu de forma positiva aos ensaios de curto circuito, sobrecarga, sobtensão e subtensão, indisponibilizando o sistema no tempo previsto em norma após o distúrbio. A estrutura física mostrou-se resistente a condições ambientais adversas (incidência de chuvas, ventos e exposição prolongada ao sol). Revelou-se necessário, porém, lonas de proteção lateral devido aos raios solares que incidem sobre os bancos, tanto no nascer quanto no pôr do sol no intuito de trazer melhor conforto para o usuário.

O local selecionado para a implantação do protótipo foi estudado conforme visto no Manual de Engenharia para Sistemas Fotovoltaicos (Pinho e Galdino, 2014) de acordo com a altura das árvores e construções ao redor, norte e sul verdadeiro e inclinação da placa de acordo com a latitude da cidade. Apesar de não ter sido deslocado para eventos fora do IFBA, 


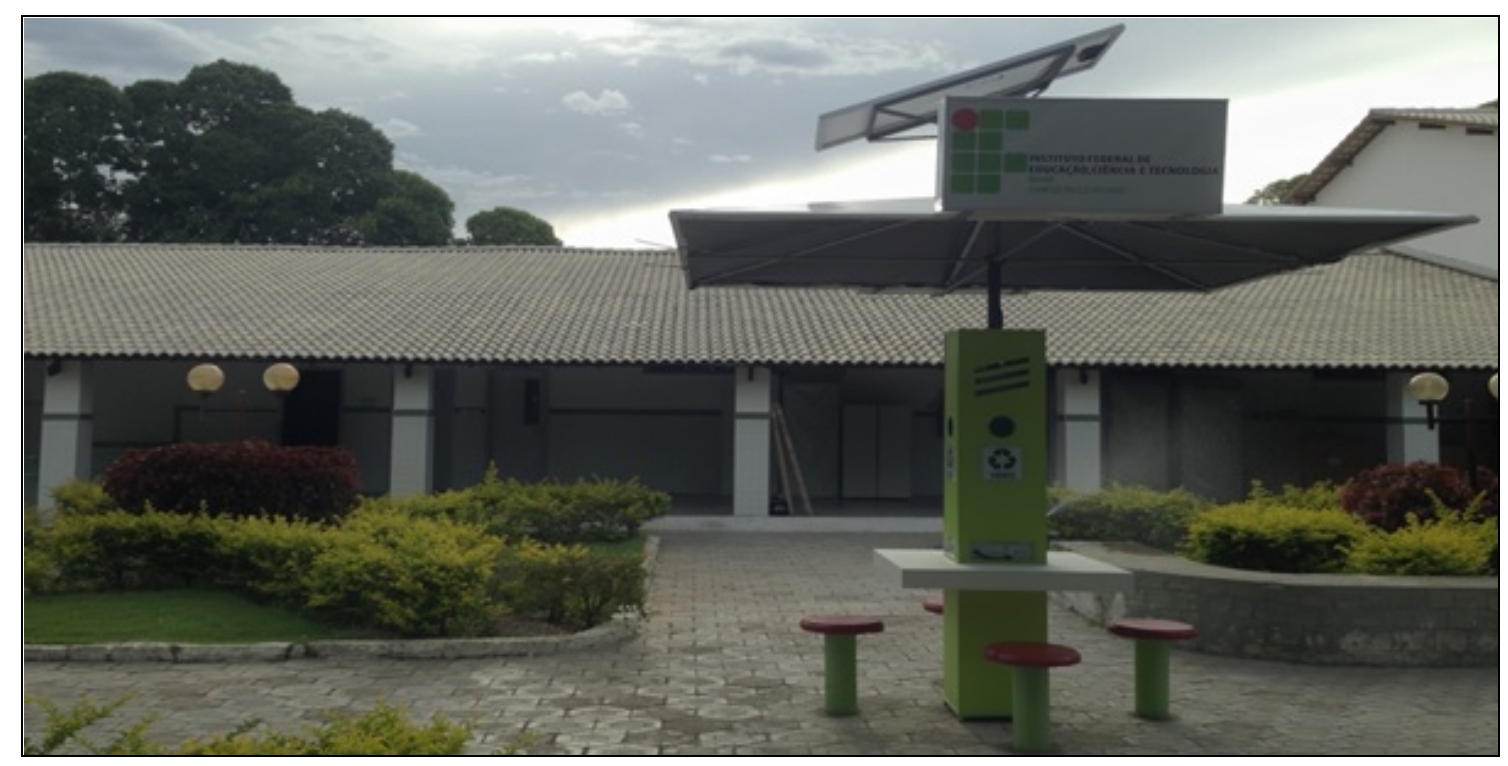

Figura 4. Protótipo finalizado e instalado no IFBA, Campus de Paulo Afonso.

Campus de Paulo Afonso, o protótipo tem notificações positivas por parte daqueles que já utilizaram.

No que tange a coleta seletiva, concretizou-se a implantação coletores para os resíduos sólidos, como: vidros, baterias, metais, papeis e plásticos. Todo o resíduo que é depositado no protótipo é colhido diariamente, ou de acordo com necessidade, e enviado mensalmente para associação de reciclagem da cidade de Paulo Afonso. Justificando-se nas características e hábitos intrínsecos ao ambiente escolar, verificouse que os materiais recicláveis com maior predominância foram latas de alumínio, papeis e plástico.

Novas etapas do projeto estão em andamento, como a quantificação dos resíduos sólidos coletados, o potencial energético em diferentes períodos do ano e pesquisas de opinião com o público atingido.

A partir da instalação do protótipo no IFBA, foram realizados e planejados treinamentos sobre o funcionamento da energia solar fotovoltaica e a importância da coleta seletiva de lixo, ainda como forma de concluir com sucesso os objetivos do projeto e difundir a cultura cientifica na instituição.

Iniciou-se então, um processo de educação ambiental que atinge toda comunidade do IFBA, Campus de Paulo Afonso, do alunado aos servidores em diversas faixas etárias. Esta comunidade vem apresentando grande aceitação ao projeto e contribuindo com sua manutenção, além de incentivar sua ampliação.

\section{Conclusão}

A criação e implantação da central de recarga de dispositivos eletrônicos pessoais à base de energia solar com coleta seletiva de resíduos integrada foi realizada com sucesso, contemplando os objetivos propostos.

O projeto também se mostrou como uma importante ferramenta para os cursos técnicos nas modalidades integrado $\mathrm{e}$ subsequente, bem como para o Curso de Bacharelado em Engenharia Elétrica em disciplinas tanto do eixo de Engenharia elétrica quanto para aquelas relacionadas à Engenharia Ambiental. É relevante mostrar que iniciativas acadêmicas podem trazer novas ideias que nos ajudem a combater a atual crise ambiental existente e a crescente demanda energética.

\section{Declaração de conflito de interesses}

Os autores declaram não haver conflitos de interesses. 


\section{Referências}

Abrelpe - Associação Brasileira de Empresas de Limpeza Pública e Resíduos Especiais. Panorama dos resíduos sólidos no Brasil (2013). São Paulo: Abrelpe, 2013. Disponível em: <http://www.abrelpe.org.br/Panorama/ panorama2013.pdf $>$. Acesso em: 25 abr. 2015.

Bittencourt, C.; Bassaco, R. L. T. Estudo do desempenho do sistema fotovoltaico conectado à rede: Estudo de caso. Curitiba: Universidade Tecnológica Federal do Paraná, 2013. (Trabalho de conclusão do Curso de Engenharia Elétrica). Disponível em: $<$ http://repositorio.roca.utfpr.edu.br/jspui/ bitstream/1/3223/1/CT_COELE_2013_2_16. pdf>. Acesso em: 25 abr. 2015.

Duham M. T.; Iverson, B. D. High-efficiency thermodynamic power cycles for concentrated solar power systems. Renewable and Sustainable Energy Reviews, v. 30, p. 758770, 2014.

NR-10 - Segurança em Instalações e Serviços em Eletricidade - Ed 2004. Disponível em: <http://www.lorencinibrasil.com.br/blog/wpcontent/uploads/2013/09/NORMAS-NR10.pdf>. Acesso em: 25 abr. 2015.

Paschoalin Filho, J. A.; Silveira, F. F.; Luz, E. G.; Oliveira, R. B. Comparação entre as massas de resíduos sólidos urbanos coletadas na Cidade de São Paulo por meio de coleta seletiva e domiciliar. Revista de Gestão Ambiental e Sustentabilidade-GeAS, v. 13, n. 3, p. 19-33, 2014. Disponível em: <http://www.revistageas. org.br/ojs/index.php/geas/article/view/208/pdf_ 1>. Acesso em: 25 abr. 2015.
Pinho, J. T.; Galdino, A. Manual de Engenharia para sistemas fotovoltaicos. Rio de Janeiro: CEPEL-CRESESB, 2014.

Silva, E. P. Estudo da viabilidade do uso de energia solar fotovoltaica no carregamento de baterias para fins diversos. Lavras: Universidade Federal de Lavras, 2010. (Trabalho de conclusão do Curso de Especialização em Formas Alternativas de Energia). Disponível em: <http://repositorio. ufla.br/bitstream/1/4527/1/TCC_Estudo da viabilidade do uso de energia solar fotovoltaica no carregamento de baterias para fins diversos>. Acesso em: 25 abr. 2015.

Silva, R. J.; Lobato, M. J. S.; Cavalcante, R. M. S.; Arrifano R. C. D.; Corrêa, F. Geração de energia elétrica sustentável, utilizando placas solares em residências, objetivando a economia de energia. Revista Engenharia Elétrica, n. 2, 8 p., 2014. Disponível em: <http://www3.iesampa.edu.br/ojs/index.php/eng_eletrica/article/ viewFile/1208/851>. Acesso em: 25 abr. 2015.

Sketchup. 3D for Everyone | SketchUp. Disponível em: <http://www.sketchup.com/>. Acesso em: 25 abr. 2015.

Soares, C. F.; Santos R. R. Exercitando a Educação Ambiental através da coleta seletiva de lixo nas escolas. Brasília: Universidade de Brasília, 2014 (Trabalho de conclusão do Curso em Educação na Diversidade e Cidadania). 
Informação da Licença: Este é um artigo Open Access distribuído sob os termos da Licença Creative Commons Attribution, que permite uso irrestrito, distribuição e reprodução em qualquer meio, desde que a obra original seja devidamente citada.

Rev. Bras. Gest. Amb. Sustent., 2015, v. 2, n. 2, p. 43-50. 